\title{
Secondary Hemophagocytic Lymphohistiocytosis
}

National Cancer Institute

\section{Source}

National Cancer Institute. Secondary Hemophagocytic Lymphohistiocytosis. NCI

Thesaurus. Code C121184.

Hemophagocytic lymphohistiocytosis due to infections, autoimmune disorders, or underlying malignancies. Signs and symptoms include fever, lymphadenopathy, hepatomegaly, splenomegaly, and pancytopenia. 